\section{¿Por qué y para qué sistematizar las experiencias de extensión universitaria?}

Oscar Jara Holliday

Director del Centro de Estudios y

Publicaciones Alforja en San José, Costa Rica. Presidente del Consejo de Educación Popular de América Latina y el Caribe (CEAAL).

2012-2016 y 2016-2020.

oscar@cepalforja.org

(iD) orcid.org/0000-0002-6309-5567
RECEPCIÓN: 24/06/19

ACEPTACIÓN FINAL: 30/07/19

\section{Resumen}

La sistematización de experiencias, como propuesta de producción de conocimiento desde las prácticas educativas, organizativas y de procesos sociales comunitarios, se ha ido convirtiendo en los últimos años en una temática de mucho interés en diversos ámbitos, y cada vez con mayor acogida en el de las experiencias de extensión universitaria. Encontramos en las universidades una preocupación creciente en torno a la necesidad de fortalecer la producción y circulación de conocimiento académico desde los proyectos, programas y actividades de extensión o acción social y sus vínculos con las dimensiones docente e investigativa.

La definición de una propuesta conceptual de sistematización de experiencias y pautas metodológicas que la hagan viable se ha ido poniendo en práctica en diversos programas de formación y capacitación, los cuales nos plantean nuevas e importantes posibilidades y desafíos.

Palabras clave: sistematización, extensión universitaria, conocimiento académico.
Cultura(s) en clave de extensión universitaria

\section{다(1)(2)(2)}

Why and what for systematize experiences of university extension?

\section{Abstract}

The systematization of experiences, as a proposal for the production of knowledge from educational, organizational and community social practices, has become in recent years a topic of great interest in various fields, and with increasing acceptance in the field of the university extension (Community, social action) experiences.

We find at the universities a growing concern about the need to strengthen the production and circulation of academic knowledge from the projects, programs and activities of extension or social action and its links with teaching and research dimensions. The definition of a conceptual proposition of systematization of experiences and methodological guidelines that make it viable, has been put into practice in various training and education programs, which pose new and important possibilities and challenges.

Keywords: systematization, university extension, academic knowledge.
Por que e para que sistematizar experiências de extensão universitaria?

\section{Resumo}

A sistematização de experiências, como proposta para a produção de conhecimento a partir de práticas educacionais, organizacionais e de processos sociais comunitários, tornou-se nos últimos anos um tema de grande interesse em diversas áreas, e com crescente aceitação nas práticas de extensão universitária.

Achamos nas universidades uma crescente preocupação com a necessidade de fortalecer a produção e a circulação do conhecimento acadêmico a partir dos projetos, programas e atividades de extensão ou ação social e suas vinculações com as dimensões de ensino e pesquisa. A definição de uma proposta conceitual de sistematização de experiências e diretrizes metodológicas que a viabilizem, tem sido colocada em prática em diversos programas de formação e educação, que apresentam novas e importantes possibilidades e desafios.

Palavras-chave: sistematização, extensão universitária, conhecimento acadêmico.

Para citación de este artículo: Jara, O. (2019). ¿Por qué y para qué sistematizar las experiencias de extensión universitaria? +E: Revista de Extensión Universitaria, 9(11), 3-9. doi: 10.14409/extension.v9i11.Jul-Dic.8675. 


\section{La sistematización de experiencias entra en el ámbito académico}

Como propuesta de producción de conocimiento desde las prácticas educativas, organizativas y de procesos sociales comunitarios, la sistematización de experiencias se ha ido convirtiendo en los últimos años en una temática relevante en muchos ámbitos tanto académicos como no académicos, en entidades públicas, en movimientos sociales, organismos no gubernamentales y agencias de cooperación internacional. Un ámbito particular en el que ha surgido un gran interés al respecto en varios países de América Latina es el de las experiencias de extensión universitaria, que también son llamadas de acción social o proyección social en diferentes universidades.

Una preocupación bastante común que encontramos en estas prácticas está relacionada con la necesidad de fortalecer la producción y circulación de conocimiento académico en las universidades desde los proyectos, programas y actividades de extensión. Pese a la reconocida y trillada afirmación de que esta es una dimensión sustantiva del quehacer universitario, al igual que la investigación y la docencia, el caso es que no es valorada de igual manera y continúa siendo muchas veces considerada como un área o una dimensión de menor importancia e incluso prescindible.

Por todo lo anterior, desde el propio campo de las personas que impulsan programas y proyectos de extensión, se analiza que es fundamental una visibilización y una valorización de ese quehacer específico, así como de sus vínculos con las dimensiones docente e investigativa, buscando romper los compartimentos estanco entre ellas para poder integrarlas efectivamente como partes componentes de un único proceso integral de formación.

En estos tiempos en que la tendencia dominante busca reforzar un enfoque mercantilista sobre las universidades públicas argumentando que lo esencial es la formación de profesionales para cubrir las necesidades del mercado y que, por lo tanto, los recursos de las universidades deberían centrarse en la tarea docente pensada como una acción cerrada a lo interno del aula, se hace necesario retomar los propósitos y principios que animaron la Reforma Universitaria de Córdoba hace más de un siglo. Como dice el reciente Manifiesto de la Universidad de Costa Rica formulado en 2018:

"Debemos reafirmar un modelo de universidad democrático y democratizador, que enfrente la desigualdad y la inequidad, así como la tendencia a la especialización de un saber cada vez más ajeno a los sectores populares y empobrecidos a los que nos debemos. A cien años de la Reforma de Córdoba, la Universidad de Costa Rica levanta la voz de nuevo, reivindicando todo el quehacer universitario, y sobre todo, la acción social o extensión como una práctica transformadora y liberadora articulada íntimamente con la docencia y la investigación. Las comunidades del futuro deberán seguir alimentando a las universidades públicas no solo con los recursos necesarios para su funcionamiento, sino con el cúmulo de saberes y conocimientos que le otorgan vida y sentido. No permitamos que la universidad vuelva a ser ese polvoriento claustro, abierto únicamente a intereses mercantiles. Luchemos por una universidad que, al contrario, haga espacio a la ternura y la esperanza de todas las personas". (AA. VV., abril de 2018:s/n)

Desde esa época en que se instaura en América Latina la extensión universitaria como componente esencial de la actividad académica e, incluso, se llevan a cabo movimientos creadores de universidades populares en América Latina, uniéndose a las experiencias 
similares que se llevaban a cabo en Europa, el modelo de universidad que se comienza a propugnar como necesario supone abrir los muros y claustros universitarios a las problemáticas más álgidas de nuestras sociedades y a la participación y propuestas de los actores sociales mayoritarios de nuestros países. De ahí que se apuesta por una universidad pública, gratuita, laica, inclusiva, comprometida socialmente y con programas de alta calidad en la extensión (o acción social), la investigación y la docencia, formando parte de un mismo y único proceso ético, político y pedagógico. La universidad como espacio de debate y confrontación de ideas. La universidad como espacio de construcción de pensamiento crítico y de relaciones solidarias.

Progresivamente, en el caso de Costa Rica, tanto desde esfuerzos interuniversitarios como han sido las propuestas de las subcomisiones de evaluación y capacitación del Consejo Nacional de Rectores (CONARE), como desde propuestas impulsadas por cada universidad en particular, en los últimos años tuvieron lugar varios procesos de formación y capacitación en teoría y metodología de sistematización de experiencias de acción social, que posibilitan a las propias personas protagonistas de esas prácticas de extensión o acción social la construcción de conocimientos académicos provenientes de ese quehacer desafiante y vivo de vínculo con comunidades y sectores sociales. ${ }^{1}$ Se procura entonces articular estos conocimientos - gracias a una labor docente crítica y comprometida - con los conocimientos y aportes provenientes de proyectos y programas de investigación, contribuyendo a la formación integral de alumnas, alumnos y de las y los propios docentes.

La formación en teoría y metodología de sistematización de experiencias de acción social tiene su punto de partida en las propias necesidades y motivaciones planteadas a sus protagonistas por los proyectos y programas que ejecutan en conjunto con personas de comunidades urbanas y rurales, así como a través de convenios con escuelas, colegios, instituciones estatales o municipales, entre otros. Se parte de la convicción de que hay una riqueza de enseñanzas en las experiencias concretas de extensión que no necesariamente estamos convirtiendo en aprendizajes ni en conocimiento académico. El activismo o la preocupación centrados en la mera ejecución de los proyectos aparece como el principal obstáculo para generar y construir esos aprendizajes, a los cuales hay que dedicar un esfuerzo reflexivo sistemático y ordenado. Claro, muchas veces el tiempo que se requiere para hacerlo no está considerado en los planes y también hacen falta orientaciones metodológicas y herramientas técnicas que lo posibiliten.

Desde ese doble punto de partida, con la convicción de que las experiencias encierran múltiples y ricos aprendizajes que es necesario identificar, descubrir y explicitar, y la necesidad de contar con orientaciones y herramientas para hacerlo, hemos ido explorando rutas posibles para sistematizar las experiencias que están marcando el rumbo de procesos de formación y capacitación que realizamos con varias universidades, tanto en Costa Rica como en otros países.

Presentamos a continuación el abordaje conceptual y metodológico con el que hemos venido trabajando, así como algunas reflexiones propositivas en torno a las pistas que se

1) En la Biblioteca Virtual de Sistematización de Experiencias del Consejo de Educación Popular de América Latina y el Caribe, CEAAL, podemos encontrar productos interesantes que han sido resultado de estos procesos: Teresita Pérez de Maza (2019). 
van abriendo en el ámbito académico respecto de la importancia y viabilidad de este enfoque de producción de conocimientos desde las prácticas.

\section{Qué entendemos por sistematización de experiencias}

Desde el punto de vista conceptual, se hace necesario diferenciar lo que se entiende comúnmente como "sistematización": ordenar, clasificar, catalogar datos e informaciones dispersas, con el concepto de "sistematización de experiencias" como interpretación crítica de los procesos vividos que, a partir de su ordenamiento y reconstrucción, posibilita identificar aprendizajes significativos que deben comunicarse y compartirse para alimentar la propia experiencia o para inspirar a otras en una perspectiva transformadora. Esta segunda acepción vinculada siempre a "experiencias" implica un ejercicio intelectual de múltiples tareas: registro, descripción, reconstrucción, análisis, síntesis, interpretación, comunicación. En definitiva, realizar un proceso de teorización a partir de las experiencias vividas que exige un procedimiento riguroso y, por eso, sistemático.

Los procesos de formación en teoría y metodología de la sistematización de experiencias que hemos estado impulsando en varios lugares se realizan desde un enfoque de educación popular, es decir, desde una perspectiva educativa liberadora, horizontal, dialógica, que busca construir un conocimiento transformador y desarrollar capacidades para el cambio social y personal a partir de ello. Supone un ejercicio de teorización desde la práctica comprometido con procesos transformadores en los cuales se refuerza nuestro quehacer como sujetos protagonistas de una historia a construir, basándonos en los intereses, necesidades, aspiraciones y propuestas de las mayorías populares que sufren asimetrías en nuestras sociedades. Por eso, las sistematizaciones de experiencias de extensión universitaria tienen un doble signo de compromiso con los sectores oprimidos, marginados, excluidos o discriminados de nuestras sociedades: por un lado, porque estos programas y proyectos significan compromiso universitario con esas problemáticas para buscar contribuir a cómo superarlas y porque sistematizarlos para generar aprendizajes significativos fortalece la comprensión crítica y propositiva por parte de las personas que son los sujetos participantes. Esto último lleva a promover un diálogo entre los saberes populares y los conocimientos académicos, que se entrelazan en los procesos de extensión o acción social dinamizando, a la vez, la dimensión investigativa y posibilitando enriquecer la dimensión docente.

Algunos aspectos claves de la concepción con la que trabajamos² son:

- Ubica la sistematización de experiencias como una interpretación que tiene como base el ordenamiento y reconstrucción de lo acontecido. Es decir que es el resultado de un esfuerzo complejo de ubicación, descripción, narración, clasificación de elementos, análisis y reflexión en torno a la experiencia vivida.

- En esta interpretación se busca identificar la lógica del proceso: dónde, cómo y por qué los distintos factores de la experiencia se relacionaron de determinada manera a lo largo de la experiencia; cuáles fueron los factores más activos y determinantes y cuáles los 
más dependientes o secundarios: qué continuidades, discontinuidades, contradicciones y rupturas se dieron en el proceso y por qué se dieron. Qué fases o etapas ha tenido la experiencia y por qué fue posible pasar de una a otra.

- La interpretación produce conocimientos críticos y aprendizajes significativos desde la particularidad de lo vivido en las experiencias y por parte de quienes las han vivido. Esto implica, muchas veces, que logremos percibir dimensiones y perspectivas inéditas que estaban presentes en nuestras experiencias pero no las habíamos percibido ni reconocido. - La sistematización de experiencias permite —entonces-apropiarnos críticamente del sentido de la experiencia, no porque este existiera de antemano sino como un "hacer nuestro" el sentido de nuestra práctica. Por ello viene a ser una construcción crítica y consciente del sentido de la experiencia, lo que posibilita no solo comprenderla en sus fundamentos y tramas invisibles sino también darnos pistas para su orientación transformadora hacia el futuro.

Lo anterior implica que reafirmamos que somos sujetos de la historia y no simplemente objetos que actuamos en ella. La sistematización de experiencias se convierte en condición de posibilidad para que comprendamos teóricamente — con un nivel de abstracción y generalización mayor- la particularidad de las experiencias de extensión y podamos asumirlas en nuestras manos con una intencionalidad transformadora.

Por ello es que la sistematización de experiencias de extensión universitaria puede contribuir a reforzar el compromiso transformador del quehacer universitario, aportar a la producción de un conocimiento académico nutrido de la riqueza de la realidad económica, social, política y cultural en la que la universidad está inserta, y a los procesos de cambio social que tengan como protagonistas a los sectores sociales de las comunidades con las que se trabaja, apoyando proyectos de gestión local y de incidencia social de alcances diversos.

Esta concepción de sistematización de experiencias rompe con tradicionales esquemas de intervención extensionista en los que se pretendía "llevar el saber académico a las comunidades" para más bien construir un conocimiento académico nutrido del diálogo de saberes producido en las experiencias. De ahí que se exija también una metodología coherente con esta visión.

\section{Una propuesta metodológica flexible y viable para hacer posible la sistematización}

En los procesos de formación en sistematización de experiencias de extensión universitaria que hemos coordinado, se han ido siguiendo los momentos (no "pasos" en un sentido lineal, ni de aplicación de receta) de una propuesta metodológica que, partiendo de esa experiencia vivida, en primer lugar, formula un plan de sistematización en el que es importante:

a) Delimitar el objeto o experiencia a sistematizar (en tiempo y lugar), identificando la práctica concreta, sus actores y condiciones, y el período que servirá de base para construir los aprendizajes.

b) Definir un objetivo preciso a alcanzar como resultado de esta sistematización.

c) Precisar un eje en torno a los aspectos centrales de la experiencia que nos interesan más y, por tanto, se convierten en un hilo conductor para leer la experiencia.

d) Identificar las fuentes de información con que se cuenta: los registros documentales, 
fotográficos, sonoros, audiovisuales, etc., y los que habría que conseguir para abordar un conocimiento a fondo del proceso de la experiencia transcurrida.

e) Formular los procedimientos, técnicas y actividades a realizar y determinar responsabilidades, fechas y productos, así como prever un presupuesto que permita solventar todo el proceso de sistematización.

Una vez elaborado el plan, viene toda una etapa de recuperación del proceso vivido, de mirar la experiencia como proceso utilizando los registros y fuentes, de identificar sus etapas, actores, interrelaciones. Para ello habrá que ordenar la información y hacer una reconstrucción histórica con base en el eje de sistematización formulado.

Esta recuperación del proceso es el sustento para hacer entonces toda una labor de análisis de diferentes aspectos por separado y realizar síntesis e interrelaciones entre los elementos encontrados. Es el momento clave y sustantivo de la interpretación crítica del proceso vivido. Allí, normalmente, surgen hallazgos de diverso tipo que no habíamos percibido cuando ocurrieron o que ahora son visibles por haber relacionado aspectos diversos que sucedieron durante la experiencia. Este momento, que se caracteriza por posibilitar una "toma de distancia" sobre lo vivido para mirarlo críticamente y que nos permite tener un mirar panorámico y de conjunto, crea las condiciones para ya no solo hacer una descripción o una narración de lo ocurrido, sino para construir una interpretación de fondo. De esta manera se abre - desde la reconstrucción del proceso vivido- un momento específico de teorización, de abstracción, de comprensión de causas, factores comunes y diferentes, tensiones y contradicciones que marcaron dicho proceso y lo definieron. Ello nos posibilita derivar, por tanto, hacia la formulación de conclusiones, aprendizajes y recomendaciones.

Finalmente, estas formulaciones deben ser comunicadas, compartidas, puestas a debate para generar consensos y orientaciones, para plantear propuestas y líneas de acción para el futuro. Aquí es donde la sistematización de experiencias hace factible no solamente "apropiarse de la experiencia pasada" sino apuntar a "apropiarse del futuro" orientando con mayor capacidad y proyección lo que se plantea y recomienda hacer.

Siguiendo esta ruta a través de talleres presenciales y ejercicios individuales y colectivos orientados virtualmente a través de una plataforma informática educativa (Moodle), con apoyo de materiales de lectura y audiovisuales, cada equipo o persona que inició el proceso algunos meses antes planteándose el interés y necesidad por sistematizar una determinada experiencia de acción social, concluye con la redacción de un artículo académico que sintetiza los resultados de su proceso de sistematización. También, a partir de todo lo realizado en esta sistematización de la experiencia, algunos equipos o personas, utilizando las fotografías, grabaciones, vídeos y matrices de recuperación del proceso, elaboran otro tipo de productos comunicativos, sea para compartirlos con colegas que trabajan en proyectos de extensión o acción social o para compartirlo con las personas de las comunidades con las que se trabajó u otras con experiencias semejantes.

Cada producto de sistematización supone un intenso trabajo de ordenamiento, reflexión, aprendizaje e intercambio. Muchos son elaborados también con las personas de las comunidades con las que se ha venido trabajando, de tal forma que sus voces y sus saberes se expresan y comparten por medio de ellos. 


\section{Pistas que van abriendo nuevas rutas}

Pese a que ya hay un camino andado de varios años en este campo, cada nueva experiencia de formación genera aprendizajes diferentes, aborda problemáticas innovadoras y exige variables metodológicas adecuadas a condiciones inéditas, así como profundizar en nuevas temáticas. De esta manera, poco a poco se van superando las tendencias al activismo que no dejan tiempo para la reflexión, a la elaboración de informes puramente descriptivos y narrativos que no incorporan reflexiones críticas, a la distancia entre el diálogo de saberes que se origina en las experiencias de extensión y los conocimientos producidos en procesos investigativos. Asimismo, es creciente la cantidad de proyectos de extensión que incorporan desde su planeación momentos específicos y recursos humanos y materiales destinados a la sistematización de las prácticas como ejercicio interpretativo crítico estrechamente vinculado a la marcha de los proyectos y no como una tarea externa, puntual o posterior.

Cada nueva experiencia de formación y capacitación en sistematización de experiencias de extensión es, en realidad, un incentivo y una oportunidad maravillosa de aprendizaje para quienes las estamos proponiendo. Seguimos, por ello, aprendiendo y compartiendo aprendizajes como los que hemos querido abordar en este artículo. Esperamos que sea también un incentivo para incorporar esta dimensión al quehacer extensionista, no como una actividad aislada, sino como un ejercicio permanente que contribuya a reforzar los esfuerzos por una universidad comprometida y coherente frente a los desafíos de nuestras sociedades.

\section{Referencias bibliográficas}

AA. VV. (abril de 2018). Manifiesto de la Universidad de Costa Rica en defensa de la universidad pública y por una acción social transformadora, en conmemoración de los 100 años de la Reforma de Córdoba. San José. Jara, O. (2012). La Sistematización de Experiencias, práctica y teoría para otros mundos posibles. San José: CEP Alforja, CEAAL, Oxfam Intermon.

Maza, T. (Coord.) (2019). Valores en la extensión universitaria. Hacia la construcción de un código deontológico en el GINEx. Sistematización de los escalones epistémicos y metodológicos. Caracas: Universidad Abierta. Recuperado de http://www.cepalforja.org/sistem/bvirtual/?p=1665 (29/05/2019).

UNA (Universidad Nacional) de Costa Rica (2016-2018): Colección Democratizando los conocimientos de la extensión universitaria. 